\title{
A Photometric Study of the Newly Discovered Eclipsing Cataclysmic Variable SDSS J040714.78-064425.1
}

\author{
T. $A k^{\mathrm{A}, \mathrm{F}}, A$. Retter $^{\mathrm{B}, \mathrm{C}}, A$. Liu $^{\mathrm{D}}$, and H. H. Esenog $\breve{l} u^{\mathrm{E}}$ \\ A Istanbul University, Faculty of Science, Department of Astronomy and Space Sciences, \\ 34119 University, Istanbul, Turkey \\ B Pennsylvania State University, Department of Astronomy and Astrophysics, University Park, \\ PA 16802-6305, USA \\ ${ }^{\text {C }}$ University of Sydney, School of Physics, Sydney NSW 2006, Australia \\ D Norcape Observatory, PO Box 300, Exmouth WA 6707, Australia \\ ${ }^{\mathrm{E}}$ Istanbul University Observatory Research and Application Center, 34119 Beyazıt, Istanbul, Turkey \\ F Corresponding author. Email: tanselak@istanbul.edu.tr
}

Received 2004 August 27, accepted 2004 December 15

\begin{abstract}
We present the results obtained from unfiltered photometric CCD observations of the newly discovered cataclysmic variable SDSS J040714.78-064425.1 made during seven nights in 2003 November. We establish the dwarf nova nature of the object as it was in outburst during our observations. We also confirm the presence of deep eclipses with a period of $0.17017 \pm 0.00003 \mathrm{~d}$ in the optical light curve of the star. In addition, we found periods of $0.166 \pm 0.001 \mathrm{~d}$ and possibly also $5.3 \pm 0.7 \mathrm{~d}$ in the data. The $0.17017 \mathrm{~d}$ periodicity is consistent within the errors with the proposed orbital period of 0.165 and $0.1700 \mathrm{~d}$. Using the known relation between the orbital and superhump periods, we interpret the 0.166 and $5.3 \mathrm{~d}$ periods as the negative superhump and the nodal precession period respectively. SDSS J040714.78-064425.1 is then classified as a negative superhump system with one of the largest orbital periods.
\end{abstract}

Keywords: accretion, accretion discs — stars: individual (SDSS J040714.78-064425.1) — dwarf novae — novae, cataclysmic variables

\section{Introduction}

SDSS J040714.78-064425.1 (hereafter abbreviated to SDSS J0407-0644, $\alpha_{2000.0}=04^{\mathrm{h}} 07^{\mathrm{m}} 14^{\mathrm{s}} .78, \quad \delta_{2000.0}=$ $-06^{\circ} 44^{\prime} 25^{\prime \prime} .1$, Downes, Webbink, \& Shara 1997) is a cataclysmic variable star discovered from SDSS (Sloan Digital Sky Survey, York et al. 2000) by Szkody et al. (2003). They also obtained $V$ filter photometry and time-resolved spectroscopy of the system while its out-of-eclipse magnitude was about $17.2 \mathrm{mag}$. According to Szkody et al. (2003), SDSS J0407-0644 is a deeply eclipsing cataclysmic variable with an eclipse depth of about 2 mag. Their photometric data revealed an orbital period of about $3.96 \mathrm{~h}$ and a strong orbital hump modulation interpreted by a prominent hot spot. However, their observations only cover $9.42 \mathrm{~h}$, i.e. two eclipses, so the error in this orbital period determination is quite large. Spectra of SDSS J0407-0644 show the double-peaked Balmer line profile during the out-of-eclipse phases, typical of accretion discs. The blue peak is stronger during the hump at phase 0.9, and the Balmer emission is unchanged during the eclipse itself (phase 0.0).

B. Monard (2004, private communication) reported that the star showed several normal dwarf nova outbursts since 2003 July. His observations also indicated two possible superoutbursts around 2003 July 19 and October 17, suggesting that the system may be classified as an SU UMa type dwarf nova candidate. A. Henden (2004, private communication) reported outbursts as well.

The possibility that SDSS J0407-0644 is an SU UMa system with an orbital period above the period gap inspired us to observe this system in an outburst and to look for superhumps.

In this paper, we present extensive photometric observations of SDSS J0407-0644, which suggest a refined orbital period, a negative superhump period, and possibly a precession period as well.

\section{Observations}

Photometric observations of SDSS J0407-0644 were made by one of us (A. L.) with a 0.3-m f/6.3 telescope coupled to an ST7 NABG CCD camera. The telescope is located in Exmouth, Western Australia, and no filter was used. Exposure times were $60 \mathrm{sec}$ every $120 \mathrm{sec}$. SDSS J0407-0644 was observed on the nights of 2003 November 5, 6, 7, 8, 9, 11, and 12. The observational log is given in Table 1. The observations span seven nights (48 hours in total).

We estimated differential magnitudes with respect to GSC4731-0533 ( $V=14.30 \mathrm{mag}$, the comparison star, $2.1^{\prime} \mathrm{N}$ of the variable), using another star (1.57' SE of 
Table 1. Journal of photometric observations made in 2003 November

\begin{tabular}{lccccc}
\hline Day & $\begin{array}{c}\text { HJD Start } \\
\text { (HJD-2452900) }\end{array}$ & $\begin{array}{c}\text { Duration } \\
(\mathrm{h})\end{array}$ & $N^{1}$ & $\begin{array}{c}\text { Mid-eclipse } \\
(\text { HJD-2452900) }\end{array}$ & $\begin{array}{c}m_{\text {out }}^{2} \\
(\mathrm{mag})\end{array}$ \\
\hline 05 & 49.09812 & 6.3 & 168 & 49.15016 & 15.48 \\
06 & 50.08346 & 6.7 & 185 & 50.32062 & 15.62 \\
& & & & 50.34176 & 15.70 \\
07 & 51.05323 & 7.5 & 207 & 51.19289 & 15.92 \\
08 & 52.08026 & 6.8 & 184 & 51.36300 & 16.06 \\
09 & 53.07978 & 6.8 & 170 & 53.21371 & \\
11 & 55.07301 & 6.8 & 180 & 55.23440 & 17.11 \\
& & & & 55.27290 & \\
12 & 56.06455 & 7.1 & 193 & 56.12916 & \\
& & & & 56.29833 & \\
\hline
\end{tabular}

${ }^{1}$ Number of observations.

${ }^{2}$ Mean out-of-eclipse magnitude.

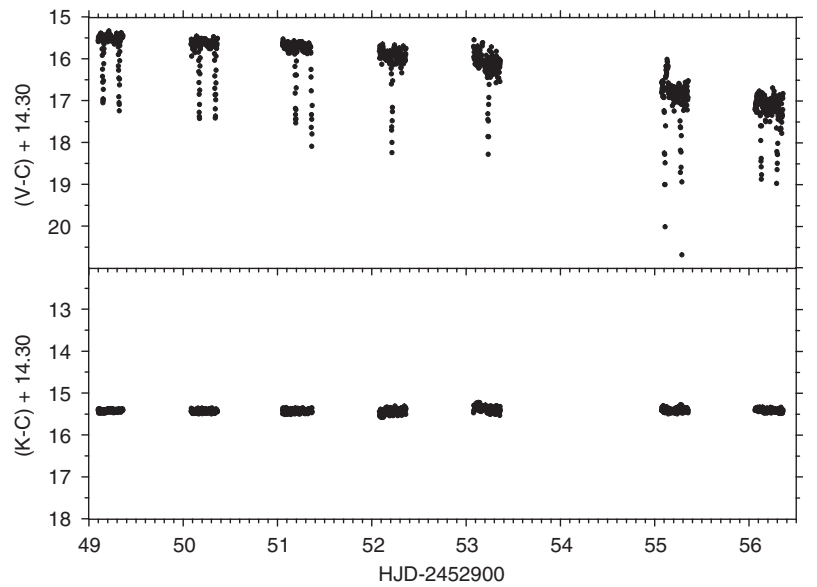

Figure 1 The light curve of SDSS J0407-0644 during the observing run. $V, C$, and $K$ represent the unfiltered magnitude of the variable, comparison, and check stars, respectively. The mean GSC magnitude of the comparison star was added to the differential magnitudes to give a rough estimate of the visual magnitude of SDSS J0407-0644 and the check star.

SDSS J0407-0644) in the field, which is not listed in the Guide Star Catalog (GSC), as the check star. The unfiltered magnitude of the check star (16.60 mag) was derived from the SBIG CCDOPS software that came with the camera.

Differential magnitudes were calculated using aperture photometry. The mean GSC magnitude of the comparison star was added to the differential magnitudes to give a rough estimate of the visual magnitude. The light curve of SDSS J0407-0644 obtained during the observations is shown in Figure 1. A part of the light curve of SDSS J0407-0644 observed on 2003 November 5 is presented in Figure 2. The observational errors were estimated from the deviations of the $K-C$ magnitudes from the nightly means and are typically about $0.03 \mathrm{mag}$.

As can be seen in Figure 1, the mean out-of-eclipse unfiltered magnitude of SDSS J0407-0644 decreased from 15.48 to $17.11 \mathrm{mag}$ during the observing run.

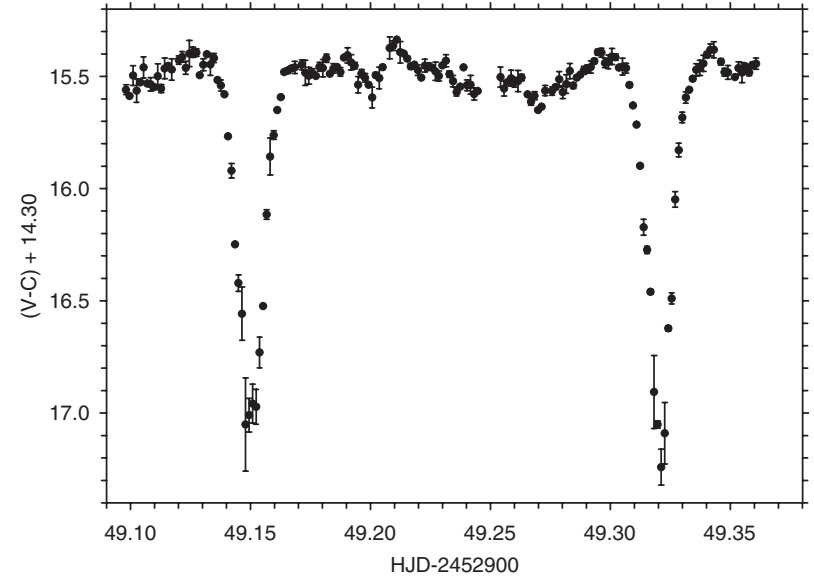

Figure 2 A part of the light curve of SDSS J0407-0644 observed on 2003 November 5.

B. Monard (2004, private communication) found the system in possible superoutbursts on 2003 July 19 and October 17, while its mean out-of-eclipse magnitudes were about 15.15 and 14.98 , respectively. However, the out-of-eclipse magnitude of SDSS J0407-0644 was about 17.7 in the observations of Szkody et al. (2003) on 2002 November 14 (see their Figure 10). These values show that we observed the system declining from an outburst, while Szkody et al. (2003) studied SDSS J0407-0644 in quiescence.

\section{Analysis}

\subsection{The Periodogram Analysis}

The period analysis was performed using the Data Compensated Discrete Fourier Transform (DCDFT, Ferraz-Mello 1981; Foster 1995), including the CLEAN algorithm (Roberts, Lehar, \& Dreher 1987). The DCDFT method is based on a least-squares regression on two trial functions, $\sin (f t)$ and $\cos (f t)$, and a constant. Here $f$ denotes the frequency. In the period analysis, we assume that the 


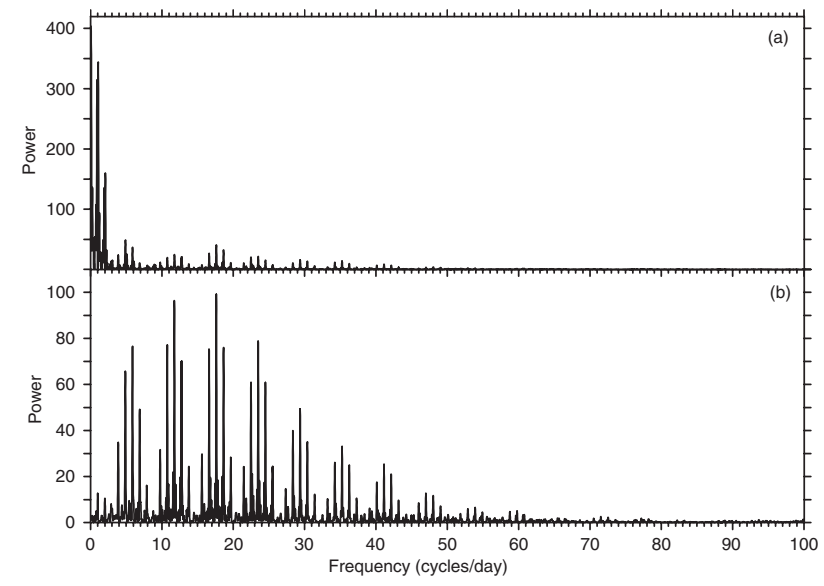

Figure 3 Power spectra of SDSS J0407-0644. (a) The power spectrum of the raw light curve. (b) The power spectrum after the mean magnitudes of the single nights were subtracted from the observations.

frequency, say $f_{1}$, that corresponds to the highest peak in the power spectrum is real and subtract it from the data. Then we find the highest peak, say $f_{2}$, in the power spectrum of the residuals, subtract $f_{1}$ and $f_{2}$ simultaneously from the raw data and calculate a new power spectrum, until the strongest residual peak is below a given cutoff level. To select the peaks, we followed a conservative approach which is similar to the method described by Breger et al. (1993) who gave a good criterion for the significance of a peak in the power spectrum. In Breger et al.'s (1993) method, the peaks in the power spectrum which are higher than the signal-to-noise ratio $(S / N)$ of 4.0 for the amplitude are indicators of real signals. In order to assign a confidence level to the power spectra, we calculated the standard error $(\sigma)$ of the power values between the frequencies for which no strong peaks appear. We assumed $4 \sigma$ to be the confidence level for the power and considered only those peaks of the power spectrum whose power was above this level. We then applied the CLEAN algorithm to remove the false peaks until the strongest residual peak was below the calculated confidence level. Note that we also searched for periodic brightness modulations by PERIOD98 (Sperl 1998), which is based on a least-squares regression on a trial function, $\sin (f t)$, along with a zero point. We found very similar power spectra from both techniques. We calculated the error in frequency from the half width at the half maximum of the peak, which is a good rough estimator of the uncertainty in frequency.

\subsubsection{The Raw Data}

As can be seen in Figure 1, the mean out-of-eclipse magnitude of SDSS J0407-0644 changed during the observing run. This magnitude change creates a strong low frequency signal at the frequency $0.046 \pm 0.002 \mathrm{c} / \mathrm{d}(21.8 \pm 1.1 \mathrm{~d})$ in the power spectrum. Thus, the power spectrum of the light curve of SDSS J0407-0644 is dominated by the aliases of this low frequency signal and the harmonics of the orbital frequency near $6 \mathrm{c} / \mathrm{d}$ as demonstrated in Figure 3. Since

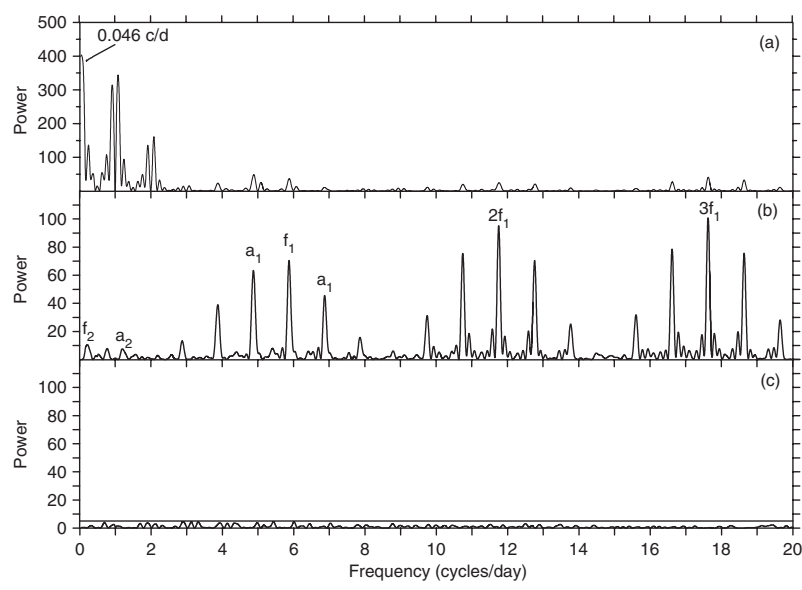

Figure 4 Power spectra of SDSS J0407-0644 zoomed into the 0$20 \mathrm{c} / \mathrm{d}$ range of frequencies. $a_{i}(i=1-2)$ represent $1 \mathrm{~d}^{-1}$ aliases of $f_{i}$. (a) The power spectrum of the raw light curve of SDSS J0407-0644. (b) The power spectrum after fitting and subtracting the very low frequency signal of $0.046 \mathrm{c} / \mathrm{d}$ that originates from the general trend of the data. (c) The cleanest power spectrum after fitting and subtracting $f_{1}=5.868$ (with its harmonics) and $f_{2}=0.19 \mathrm{c} / \mathrm{d}$. The confidence level of $5.08(4 \sigma)$ for the power is shown with a horizontal line.

the observations were done from a single site, the $1 \mathrm{c} / \mathrm{d}$ alias is also very strong, as expected.

After fitting and subtracting the very low frequency signal of $0.046 \mathrm{c} / \mathrm{d}$ that originates from the general trend of the light curve, the orbital frequency and its harmonics at the frequencies $11.753 \pm 0.009$ and $17.628 \pm 0.009 \mathrm{c} / \mathrm{d}$ dominate the power spectrum of the residuals as shown in Figure 4b. The peak that corresponds to the orbital modulation is found at the frequency $f_{1}=5.868 \pm 0.009 \mathrm{c} / \mathrm{d}$ $(0.1704 \pm 0.0003 \mathrm{~d})$. In order to find a confidence level for the power, we calculated the standard error of the power level to be 1.27 between 8 and $20 \mathrm{c} / \mathrm{d}$ after subtracting the harmonics of $f_{1}$. By considering this standard error as the noise level, we calculated the confidence level to be $4 \sigma=5.08$ for the power, as described above. To search for additional signals, $f_{1}$ and its harmonics were also subtracted from the data. In the power spectrum of the residuals, the strongest peak corresponds to the frequency of $f_{2}=0.19 \pm 0.02 \mathrm{c} / \mathrm{d}(5.3 \pm 0.7 \mathrm{~d})$. The amplitude of this signal is $0.16 \pm 0.03 \mathrm{mag}$. After removing $f_{2}$ from the data, we concluded that there is no additional significant signal in the light curve, since the power level in the residual power spectrum (Figure 4c) is lower than the confidence level. However, the presence of the $5.3 \mathrm{~d}$ is very questionable for the following reasons. (1) Since the data cover about $7.3 \mathrm{~d}$, we only have less than 1.5 cycles of the proposed $5.3 \mathrm{~d}$ period. (2) We subtracted the general trend of the light curve which may affect low frequencies; thus, this low frequency signal can be an artifact of the removal of the $0.046 \mathrm{c} / \mathrm{d}$ signal. (3) For a decaying system one may expect low frequency signals.

\subsubsection{The Frequencies near 4-8 c/d}

In order to test the significance of the peak found at $5.868 \mathrm{c} / \mathrm{d}$ and to search for additional signals near this 


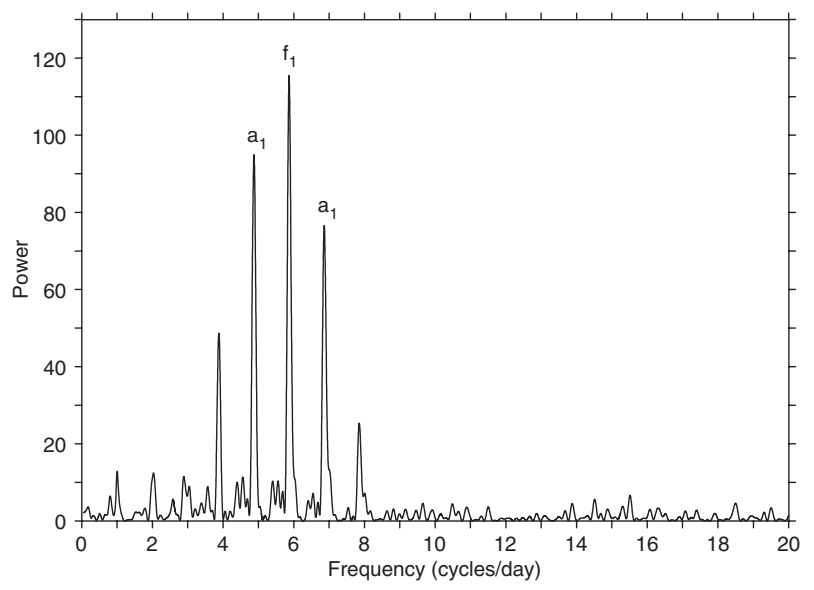

Figure 5 The power spectrum of the light curve of SDSS J04070644 zoomed into the $0-20 \mathrm{c} / \mathrm{d}$ range after the mean out-of-eclipse magnitudes of each night were subtracted from the observations. The $\geq 2$ harmonics of $f_{1}$ were removed from the power spectrum for clarity. $a_{1}$ represent $1 \mathrm{~d}^{-1}$ aliases of $f_{1}$.

frequency, we calculated the mean magnitudes of each night and subtracted them from the observations of SDSS J0407-0644.

The power spectrum of this de-trended data is dominated by the harmonics of the orbital frequency and the $1 \mathrm{c} / \mathrm{d}$ aliases which originate from the observational gap. The harmonics in the power spectrum are the result of the non-sinusoidal shape of the eclipses. The power spectrum is shown in Figure 5. In this power spectrum, the peak that corresponds to the orbital modulation is found at the frequency $5.869 \pm 0.009 \mathrm{c} / \mathrm{d}(0.1704 \pm 0.0003 \mathrm{~d})$ which is consistent with $f_{1}$ found above. By considering the standard error $\sigma=1.15$ of the power between 8 and $20 \mathrm{c} / \mathrm{d}$ as the noise level after subtracting the harmonics of $f_{1}$, we calculated the confidence level to be $4 \sigma=4.60$ for the power.

To search for additional signals, $f_{1}$, its harmonics, and the $1 \mathrm{c} / \mathrm{d}$ aliases were subtracted from the data. The strongest peak in the power spectrum of the residuals corresponds to the frequency of $f_{3}=6.03 \pm 0.05 \mathrm{c} / \mathrm{d}$ $(0.166 \pm 0.001 \mathrm{~d})$ which is demonstrated in Figure $6 \mathrm{a}$. The amplitude of this signal is $0.04 \pm 0.02 \mathrm{mag}$. It can be seen in Figure $6 a$ that the power level of this very weak peak is just above the confidence level. The light curve folded on the 0.1704 and $0.166 \mathrm{~d}$ periods is shown in Figures $8 \mathrm{a}$ and $8 \mathrm{~b}$, respectively.

\subsubsection{The Out-of-Eclipse Data}

Since the significance of the signal with the frequency $f_{3}=6.03 \mathrm{c} / \mathrm{d}$ is questionable due to its low amplitude in the power spectra of the light curve, we removed the eclipses by hand in the de-trended data in order to test the presence of this signal.

Power spectra of the out-of-eclipse data are displayed in Figure 7. In the power spectrum, we find the strongest peak at the frequency $6.92 \pm 0.02 \mathrm{c} / \mathrm{d}$ which is near the $1 \mathrm{~d}^{-1}$ alias of the orbital frequency $5.869 \mathrm{c} / \mathrm{d}$. The $1 \mathrm{~d}^{-1}$

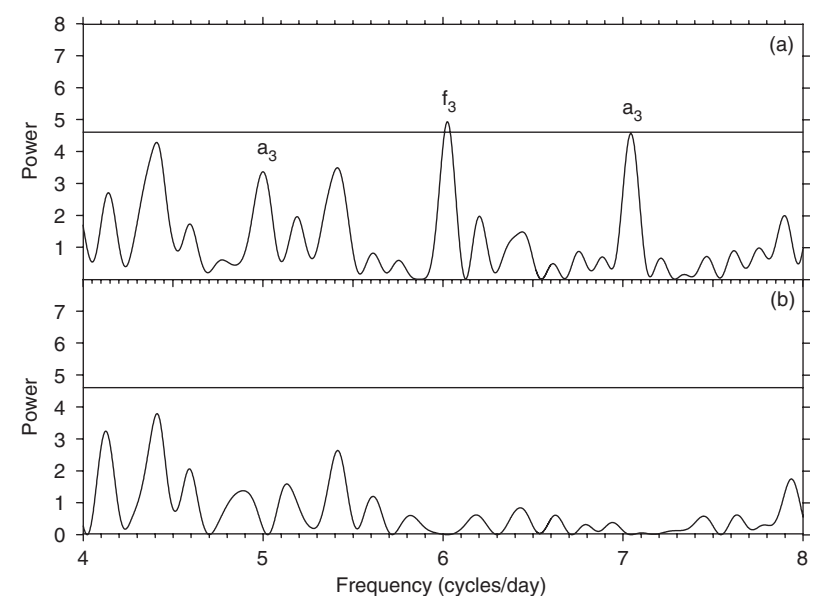

Figure 6 The power spectra of the light curve of SDSS J04070644 zoomed into the interval $4-8 \mathrm{c} / \mathrm{d}$. The confidence level of 4.60 $(4 \sigma)$ is shown with horizontal lines. $a_{3}$ represent $1 \mathrm{~d}^{-1}$ aliases of $f_{3}$. (a) The power spectrum after $f_{1}$ and the $1 \mathrm{c} / \mathrm{d}$ alias are removed. (b) The power spectrum after fitting and subtracting $f_{3}=6.03 \mathrm{c} / \mathrm{d}$ as well.

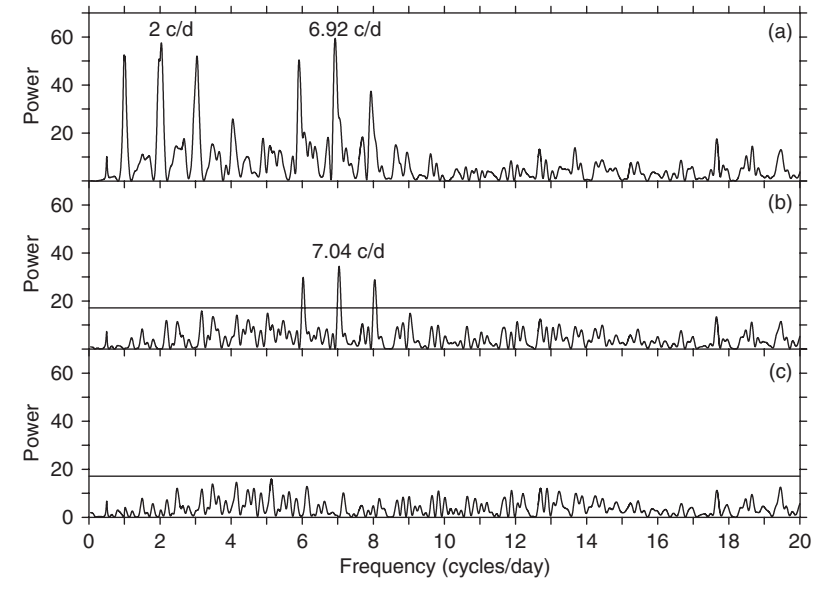

Figure 7 The power spectra of the light curve of SDSS J04070644 zoomed into the $0-20 \mathrm{c} / \mathrm{d}$ range of frequencies after removing the eclipses by hand. The confidence level of $17.16(4 \sigma)$ is shown with horizontal lines. (a) The power spectrum of the data. (b) The power spectrum of the residuals after fitting and subtracting the peaks that correspond to the $1 \mathrm{~d}^{-1}$ aliases of the orbital frequency $(6.92 \mathrm{c} / \mathrm{d})$ and the observational gaps $(2 \mathrm{c} / \mathrm{d})$. (c) The power spectrum of the residuals after fitting and subtracting the peak at $7.04 \mathrm{c} / \mathrm{d}$ as well.

alias of the frequency that corresponds to the observational gaps is also very prominent. Finding the strongest peaks at the frequencies that correspond to the $1 \mathrm{~d}^{-1}$ aliases of the signals present in the data is not a surprise since we changed the window function of the data by removing the eclipses. After subtracting the $1 \mathrm{~d}^{-1}$ aliases of the orbital frequency and the observational gaps, we find the strongest peak in the power spectrum of the residuals at the frequency $7.04 \pm 0.02 \mathrm{c} / \mathrm{d}$, which is consistent with the $1 \mathrm{~d}^{-1}$ alias of the candidate signal at the frequency $f_{3}=6.03 \mathrm{c} / \mathrm{d}$. Thus, we conclude that the $f_{3}=6.03 \mathrm{c} / \mathrm{d}$ signal is indeed present in the light curve. In Figure $7 \mathrm{~b}$, the standard error $\sigma$ of the power in the frequency interval $0-20 \mathrm{c} / \mathrm{d}$ is 4.29 . 


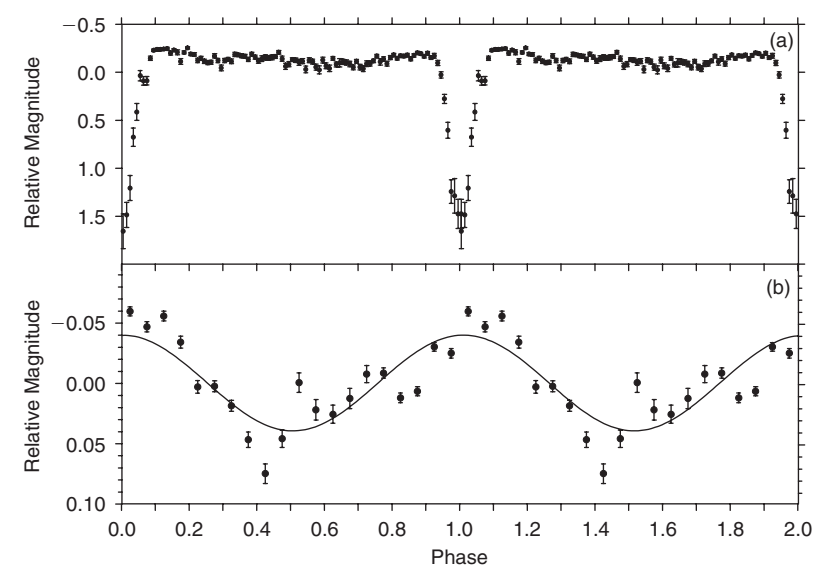

Figure 8 The folded light curves. The data used here are the observations after the nightly means were subtracted (see Section 3.1.2). Error bars represent one standard deviation of the mean values. (a) The light curve folded on the $0.1704 \mathrm{~d}$ period and binned into 100 bins. (b) The light curve folded on the $0.166 \mathrm{~d}$ period and binned into 20 bins after the eclipses were removed by hand. The solid line shows the sine fit.

By considering $\sigma$ as the noise level, we calculated the confidence level to be $4 \sigma=17.16$ for the power.

\subsection{The Ephemeris and Eclipse Depths}

The observations cover 12 usable eclipses. The corresponding mid-eclipse timings were determined by fitting a Gaussian to the data near the eclipses and are given in Table 1. We applied a linear least-squares fit to the minima times and found the following orbital ephemeris:

$$
T_{\min }(H J D)=2452949.1504(6)+0.17017(3) E .
$$

Since the error in this value is smaller than the result from the periodogram analysis where the error was defined as the half width of the peaks at half maximum, we adopt this value for the orbital period.

We also measured the eclipse depths by fitting a Gaussian to the eclipse profiles after we subtracted the mean magnitudes of each night from the observations. The eclipse depths given in Szkody et al. (2003) were about $1.5 \mathrm{mag}$. However, the mean eclipse depth is $1.80 \pm 0.24 \mathrm{mag}$ in our observations and they range between 1.5 and 2.2 mag.

\section{Discussion}

The $0.17017 \mathrm{~d}$ period that we found in the data is consistent with the orbital period ( $0.165 \mathrm{~d})$ suggested by Szkody et al. (2003) who did not give an error estimation for the orbital period. Since our observing run is much longer than the study of Szkody et al. (2003) and since they used only two eclipses to determine the periodicity, while we used 12, our value is more precise. It should be noted that B. Monard (2004, private communication) reported an orbital period of $0.1700 \pm 0.0002 \mathrm{~d}$ from his observations which covered four eclipses obtained at Bronberg Observatory/CBA, Pretoria. Thus, the $0.17017 \mathrm{~d}$ period found from the photometric observations of SDSS J0407-0644 in this study is naturally explained as the orbital period of the system. In short, our data confirm the presence of eclipses in the light curve of SDSS J0407-0644 as deep as 2 mag and this implies that the orbital period is $0.17017 \mathrm{~d}$.

The $6.03 \mathrm{c} / \mathrm{d}$ frequency corresponds to the period $0.166 \pm 0.001 \mathrm{~d}$, which is $\sim 3 \%$ shorter than the suggested orbital period of $0.17017 \mathrm{~d}$. Negative superhump periods are a few per cent shorter than the orbital periods (Patterson et al. 1997; Patterson 1999, 2001; Retter \& Naylor 2000). The $0.166 \mathrm{~d}$ period fits this trend and thus can be understood as the negative superhump period in SDSS J0407-0644. Negative superhumps are explained as the beat between the orbital period and the nodal precession of the disc. In this model, the relation between the orbital period $P_{\text {orb }}$, the negative superhump period $P_{\mathrm{sh}}^{-}$, and the precession period of the disc $P_{\mathrm{pr}}$ is given by $1 / P_{\mathrm{pr}}=1 / P_{\mathrm{sh}}^{-}-1 / P_{\mathrm{orb}}$, or $f_{\mathrm{pr}}=f_{\mathrm{sh}}^{-}-f_{\text {orb }}$. By choosing $f_{\text {orb }}=5.869 \mathrm{c} / \mathrm{d}$ and $f_{\text {sh }}^{-}=6.03 \mathrm{c} / \mathrm{d}$ from the power spectrum of SDSS J0407-0644 (Section 3), we calculate a precession frequency of $f_{\mathrm{pr}}=0.16 \pm 0.04 \mathrm{c} / \mathrm{d}$. Thus, we expect a signal with the frequency of $f_{\mathrm{pr}} \approx 0.16 \mathrm{c} / \mathrm{d}$ in the light curve of SDSS J0407-0644. This precession frequency is in agreement with the signal found from the light curve at $f_{2}=0.19 \pm 0.02 \mathrm{c} / \mathrm{d}$ (Section 3). However, the presence of the $5.3 \mathrm{~d}$ period is very questionable (see Section 3.1.1).

From our results SDSS J0407-0644 can be classified as a negative superhump system with one of the largest orbital periods. Only TV Col with an orbital period of $5.5 \mathrm{~h}$ (Retter et al. 2003) and AT Cnc at $4.8 \mathrm{~h}$ (Nogami et al. 1999; Kozhevnikov 2004) have longer orbital periods and show superhumps.

Positive superhumps are also observed in cataclysmic variables. They are explained as the beat between the binary motion and the precession of the disc in the apsidal plane (Patterson 1999). The positive superhump periods are a few per cent longer than the orbital periods. Patterson (1999) found that the period deficits in the negative superhump systems are about half the period excesses in the positive superhump systems: $\epsilon_{-} \approx-0.5 \epsilon_{+}$, where $\epsilon=\left(P_{\text {sh }}-P_{\text {orb }}\right) / P_{\text {orb }}$. Retter et al. (2002) further suggested that the ratio $\epsilon_{-} / \epsilon_{+}$, which is expected to be 0.5 according to Patterson (1999), is correlated with the orbital period. We found a negative superhump period deficit of -0.026 for SDSS J0407-0644 (Section 3). From the relations between the negative superhump deficit and the positive superhump excess mentioned above, we expect a positive superhump excess of about $\epsilon_{+} \approx+0.04-0.05$ for SDSS J0407-0644. This yields a positive superhump frequency around 5.58-5.63 c/d. As can be seen in Figure 6 there is a very weak peak at the frequency $5.41 \mathrm{c} / \mathrm{d}$. However, it is below the confidence level. Thus, we conclude that the system did not show a positive superhump or that its amplitude was below the detection limit in our observations.

The mean eclipse depth of the system in our data is $1.80 \pm 0.24$ mag. Szkody et al. (2003) found an orbital period of $3.96 \mathrm{~h}$ and a strong orbital hump modulation 
in the light curve of SDSS J0407-0644 that included only two eclipses (see their Figure 10). However, the amplitude of orbital hump in our observations was about 10 times smaller than that observed by Szkody et al. (2003). We observed SDSS J0407-0644 in an outburst while Szkody et al. (2003) studied it in quiescence (Section 2). Thus, the difference between the amplitudes of the out-of-eclipse humps obtained in this study and in Szkody et al. (2003) may be due to the difference in the brightness state of the system.

These are the first determinations of the superhump and the possible precession periods in the light curve of SDSS J0407-0644. Further observations are needed to confirm our results.

\section{Acknowledgments}

We thank the anonymous referee for a thorough report and useful comments that helped improve an early version of the paper. This work was partially supported by a postdoctoral fellowship from Penn State University and by the Research Fund of the University of Istanbul, Project Numbers BYP-409/26042004 and BYP-239/0606082003.

\section{References}

Breger, M., et al. 1993, A\&A, 271, 482

Downes, R. A., Webbink, R. F., \& Shara, M. M. 1997, PASP, 109,345

Ferraz-Mello, S. 1981, AJ, 86, 619

Foster, G. 1995, AJ, 109, 1889

Kozhevnikov, V. P. 2004, A\&A, 419, 1035

Nogami, D., Masuda, S., Kato, T., \& Hirata, R. 1999, PASJ, 51, 115

Patterson, J. 1999, in Disk Instabilities in Close Binary Systems, eds. S. Mineshige, \& C. Wheeler (Tokyo: Universal Academic Press), 61

Patterson, J. 2001, PASP, 113, 736

Patterson, J., Kemp, J., Saad, J., Skilman, D. R., Harvey, D., Fried, R., Thorstensen, J. R., \& Ashley, R. 1997, PASP, 109, 468

Retter, A., \& Naylor, T. 2000, MNRAS, 319, 510

Retter, A., Chou, Y., Bedding, T. R., \& Naylor, T. 2002, MNRAS, 330, L37

Retter, A., Hellier, C., Augusteijn, T., Naylor, T., Bedding, T. R., Bembrick, C., McCormick, J., \& Velthius, F. 2003, MNRAS, 340, 679

Roberts, D. H., Lehar, J., \& Dreher, J. W. 1987, AJ, 93, 968

Sperl, M. 1998, CoAst, 111, 1

Szkody, P., et al. 2003, AJ, 126, 1499

York, D. G., et al. 2000, AJ, 120, 1579 